\title{
Long Distance Agreement and Restructuring
}

\author{
Alice Davison, University of Iowa \\ alice-davison@uiowa.edu
}

Optional Long Distance Agreement (LDA) is possible across an non-finite complement boundary. The optionality has been explained as choice between two syntactic object complements of the matrix verb (Boeckx (2004), Bhatt (2005), Bobaljik and Wurmbrand (2005)). One complement is a full TP/CP, the other a reduced complement (Wurmbrand 2001). In Hindi-Urdu the direct object of an infinitive optionally agrees with both the matrix verb and the embedded verb (1). The grammatically feminine plural object kitaabeeN 'books' determines feminine plural agreement, otherwise the verbs have the default masculine singular form.

(1) siitaa-nee [PRO yee kitaabeeN paRh-nii] caah-iiN / paRh-naa] caah-aa Sita.f.s-erg these book.f.pl read-inf.f want-pf.fpl read-inf.ms want-pf 'Sita wanted to read these books.' (Hindi-Urdu)

(2) a. [Subject $\left[{ }_{\mathrm{vP}}\left[{ }_{\mathrm{VP}}\left[{ }_{\mathrm{IP}}\left[\mathrm{vP}\left[\mathbf{P R O}\left[_{\mathrm{v}},{ }_{\mathrm{VP}} \mathrm{OBJ} \mathrm{V}\right] \mathrm{v}\right]\right]\right.\right.\right.$ Inf $\left.\left.\left.\left.^{\mathrm{o}}\right] \mathrm{V}\right] \mathrm{v}\right]\right]$ Non-restructuring b. [Subject $\left.\left.\left[_{\mathrm{vP}}\left[{ }_{\mathrm{VP}}\left[{ }_{\mathrm{IP}}\left[{ }_{\mathrm{vP}}\left[\mathrm{VP}_{\mathrm{V}} \mathrm{OBJ} \mathrm{V}\right] \mathrm{v}\right]\right] \mathrm{Inf}^{\circ}\right] \mathrm{V}\right] \mathrm{v}\right]\right] \quad$ Restructuring (Bhatt 2005: 799)

This agreement pattern is possible for other verbs such as 'know (how)', 'be obliged', 'like', 'learn how', 'allow', and one of the two verbs 'begin'; all of which resemble restructuring verbs in other languages (Wurmbrand (2001)).

Bhatt (2005) proposes that LDA is possible only with the Restructuring option (2b), which crucially lacks an embedded PRO subject. In this paper, I use data from Hindi-Urdu which have not previously been discussed in connection with the LDA issue and its association with Restructuring. My arguments are focused on showing that a subject is syntactically projected in both the LDA and non-LDA versions of sentences. There is indirect evidence for PRO in sentences like (1), as well as direct evidence for an overt nominative subject in subject complements which have LDA. My arguments are based on (a) a case restriction on PRO, (b) the binding theory, which requires a local subject for complex reflexives, and (c) the occurrence of LDA in subject complements, which are not explained by a choice of a full CP versus a reduced infinitive, as restructuring never affects subject complements.

First, Hindi-Urdu has a case restriction on PRO. There are subjects with dative lexical case (3a), contrasting with ergative case for other transitive verbs (3b)

(3) a. baccooN-koo miThaaiyaaN mil-iiN child.pl-dat sweet.f.pl get-f.pl 'The children got sweets.

b. . baccooN-nee miThaaiyaaN khaa-iiN child.pl-erg sweet.f.pl eat-f.pl 'The children ate sweets.

If the dative subject sentence (3a) is embedded as the complement of 'want' (4), the sentence is robustly ungrammatical. It would be fine if the embedded verb were 'eat' or 'take', which do not require lexical case on the local subject. This is true whether or not there is LDA $(4 a, b)$. The LDA option is shown as (4a), with restructuring.

(4) a. *baccooN-nee [PRO miThaaiyaaN mil-naa] caah-aa Non-restructuring child.pl-erg sweet.f.pl get-m.s want-pf.m.s. 
'The children wanted [ PRO to get sweets.'

\section{b. *baccooN-nee [ miThaaiyaaN mil-nii] caah-iiN Restructuring child.pl-erg sweet.f.pl get-m.s want-pf.f.pl \\ 'The children wanted [to get sweets.'}

The lexical case condition makes (4a) ungrammatical, because the PRO subject gets dative case. But if PRO is absent in (4b), in the Restructuring option for the complement, then the sentence should be good, as there is no PRO to receive lexical case. But (4b) is as bad as (4a).

A second argument for the presence of PRO in the complement infinitive is based on the coindexing conditions of the complex reflexive. Its antecedent must be a local subject. The 'permissive' or 'allow' construction has a subject an object of permission and requires LDA (Butt 1995, Bhatt 2005).

(5) maaN-nee baccooN-koo [miThaaiyaaN khaa-nee] diiN /*di-yaa mother-erg child.m.pl sweet.f.pl eat-inf-obl give.pf.f.pl/give/m.s 'Mother allowed the children [PRO to eat sweets].'

If LDA is required, then on the Bhatt hypothesis, the complement must be a reduced infinitive without PRO. But this construction shows evidence of a local subject, PRO, which binds a complex reflexive. The matrix indirect object is not a possible binder, as it is not a subject, though it controls the PRO embedded subject. Hindi/Urdu has both simple Xo and complex XP reflexives (6) both of which are subject oriented (7), and cannot be bound by an indirect object (7). Note that these sentences show default agreement, because of the obligatory postpositions on the subject, indirect object and object.

(6) $\operatorname{raadha(i)-nee~} \operatorname{apnee(i)-koo/~apnee~} \operatorname{aap}(\mathrm{i})-\mathrm{koo} / \mathrm{usee}(* \mathrm{i} / \mathrm{j})$ aaiinee $=\mathrm{meeN}$ deekh-aa Radha-erg self-dat self's self-dat her-dat mirror=in see-pf.m.s 'Radha(i) saw herself(i)/ her(*i/j) in the mirror.

(7) maaN(i)-nee raadhaa(j)-koo apnee(i)-koo/ apnee aap(i)-koo/usee(*i/j)

mother-erg Radha-dat self-dat self's self-dat her-dat aaiinee-meeN dikhaa-yaa mirror-in show-pf.ms 'Mother(i) showed Radhaa(j) herself $\left(\mathrm{i} / *_{\mathrm{j}}\right) / \operatorname{her}\left(*_{\mathrm{i}} / \mathrm{j}\right)$ in the mirror.' [Subj] [IO] [DO]

In infinitive complements, the simplex reflexive apnee-koo can be bound either by the local subject or the matrix subject (8), but in the same context, the complex reflexive apnee aap-koo may not be bound by the matrix subject (9). It can only be locally bound by PRO, a subject, as the locus of permission, the matrix indirect object, is not itself a subject (Gurtu (1992).

(8) $\operatorname{maaN}(\mathrm{i})$-nee $\operatorname{shyaam}(\mathrm{j})-\mathrm{koo}[\mathrm{PRO}(\mathrm{j})$ apnee $=\mathbf{k o o}(\mathbf{i} / \mathbf{j}) \quad$ Simplex, ambiguous mother-erg Shyam-dat self's-dat / self's gumnaam patr bheejnee]-see manaa kiyaa anonymous letters send-inf--from forbidden do-pf 'Mother(i) forbade Shyam(j) [PRO(j) to send self(i/j) anonymous letters].' apnee $(\mathbf{i} / \mathbf{j})=$ 'Mother, Shyam'. (Davison 2001)

(9) maaN(i)-nee shyaam(j)-koo [PRO(j) apnee aap(*i/j)-koo Complex, unambiguous mother-erg Shyam-dat self's self-dat gumnaam patr bheejnee]-see manaa kiyaa 
anonymous letters send-inf--from forbidden do-pf

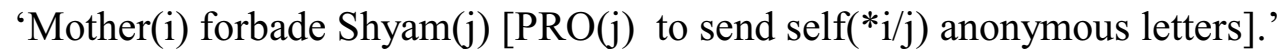

apnee $\operatorname{aap}(* \mathbf{i} / \mathbf{j})=$ 'Shyam $/{ }^{*}$ Mother' (Davison 2001)

The same binding relations are found in the 'allow' construction (10) and (11), which is supposed to be an obligatory restructuring verb, on the basis of its obligatory LDA in (5).

(10) maaN(i)-nee raadhaa(j)-koo [PRO(j) apnee (i/j)-koo deekh-nee

mother-erg Radhaa-dat self's self=dat see-inf-obl not

nahiiN diyaa

give-pf.ms

'Mother(i) did not allow Radha(j) [PRO(j) to look at self(i/j)]]. Simplex reflexive, ambiguous

(11) maaN(i)-nee raadhaa(j)-koo [PRO(j) apnee aap(*i/j)-koo deekh-nee]

mother-erg Radhaa-dat self's self-dat see-inf-obl not

nahiin diyaa

give-pf.ms

'Mother(i) did not allow Radha(j) [PRO(j) to look at self's self(*i/j)].' Complex. unambiguous

The local reading in (11) is explained if there is object control of embedded PRO. This is in indirect argument, as the sentences with binding relations do not show LDA, and the sentences with obligatory LDA do not have complex anaphors.

Finally, there are LDA sentence with subject infinitives. The infinitive subject is overt and has either genitive case (12) or nominative case (13). Nominative case is possible if the subject has inanimate reference

(12) [basooN -kaa Diipoo-see nikhal-naa $]$ shuruu hu-aa Default bus.f. pl.obl gen depot-from come.out-inf.ms beginning be-pf.ms

'Buses began to leave the station.' (K.V. Subbarao, p.c.)

(13) $[$ baseeN Diipoo-see nikhal-nii] shuruu hu-iiN LDA bus.f. pl.[Nom] depot-from come.out-inf.f s beginning be-pf.f.pl 'Buses began to leave the station.' (K.V. Subbarao, p.c.)

Just as with direct objects in the unmarked, nonpostpositional object case, there is long-distance agreement of nominative subjects of infinitive clauses. Restructuring, or reduced complements, is known to occur only for object complements, so the restructuring explanation cannot account for the LDA in (13).

I have argued that PRO is always projected in LDA structures. Unlike the corresponding verbs in other languages surveyed in Wurmbrand 2001, Hindi matrix verbs like those in (14)do not select both a full TP and a reduced TP/VP complement, even though they resemble restructuring verbs in other languages (Wurmbrand (2001); Wurmbrand and others note broad crosslinguistic similarities of restructuring verbs, combined with many language specific differences, as well as variations among speakers of a particular language.

(14) 'want' 'know (how), 'be obliged', 'like' 'learn (how)', 'begin', 'allow' Instead, in Hindi-Urdu, verbs select either reduced VP/TP (15) or a full TP. We can tell which select reduced complements without PRO by the fact that there is no violation of the restriction on dative subjects, as in the instance of the modal 'be able' or one of the verbs 'begin.' lagn-aa (16). These verbs, modals, implicative and aspectual verbs, select for VP or reduced TP.

(15) maaN-koo baccooN-par kroodh aa nahiiN sak-aa VP complement 
mother-dat children-on anger.m.s come not be.able-pf.m.s

'Mother couldn't get angry with the children.'

(16 us-koo bhaaii-par kroodh aa-nee lag-aa Restructuring]

3s-dat brother-on anger.m.s come-inf.obl begin-pf.m.s

'He/she began to get angry at his/her brother.'

(17) * us-nee [PRO bhaaii-par kroodh aa-naa] shuruu ki-yaa Non-RS 3s.-erg brother-on anger.ms come-inf beginning do-pf.ms.

'He/she began [PRO to get angry at his/her brother].'

us-nee [PRO kitaab $\quad$ paRh-nii]
3s-erg shuruu
'He/she began [PRO to read a book.'

In contrast, the other verb 'begin' shuruu kar-naa' is associated with both the dative restriction (17) and LDA (18). This verb selects for a full TP. The contrast is summarized in (19):

(19) a. lag-naa 'begin, lit. strike' selects reduced oblique infinitive, no PRO.

b. shuruu kar-naa 'begin, lit. do a beginning' selects a full TP with PRO.

The sentences (15) and (16) show that in Hindi-Urdu there are structurally reduced complements of modals, aspectuals and implicative verbs (cf. Wurmband 2001), which have obligatory agreement typical of monoclausal structures.). In these structures there is no evidence for PRO or other projected subject. But others, (17), (18) and the sentences discussed above with the verbs listed in (14) select a full TP with PRO Hindi-Urdu verbs have only one c-selection option, either VP or TP, and there seem not to be the kind of multiple selections which would explain LDA.

Complements in LDA sentences have projected subjects in Hindi-Urdu. Restructuring and obligatory control are therefore not necessary conditions for LDA. These results rule out an otherwise compelling class of explanation for LDA, at least in this and similar languages. Movement of the embedded direct object cannot be the whole explanation, because of the subject agreement in subject clauses in (13). The questions remain of what explains the unusual Long Distance Agreement over a non-finite boundary, and why it is optional.

\section{References}

Bhatt, Rajesh K. (2005) Long-distance agreement in Hindi-Urdu. Natural Language and Linguistic Theory 23, 757-807.

Bobaljik, Jonathan and Susi Wurmbrand (2005) The domain of agreement. Natural Language and Linguistic Theory 23, 809-865.

Boeckx, C. (2004) Long-distance agreement in Hindi: Some theoretical implications. Studia Linguistica 58, 23-36.

Davison, Alice (2001) Long-distance anaphors. In P. Cole et al. (eds) Long distance anaphors. Academic Press. Irvine: Academic Press, 47-82.

Davison, Alice (2008) A case restriction on control: implications for movement. Journal of South Asian Linguistics. Center for the Study of Language and Information. http://jsal-journal.org. Gurtu, Madhu (1992) Anaphoric relations in Hindi and English. Delhi: Munshiram Manoharlal. Wurmbrand, Suzanne (2003) Infinitives: Restructuring and clause structure. Berlin: Mouton de Gruyter. 